\title{
The role of dry-lab and cadaveric simulation for cystoscopy and intravesical Botulinum toxin injections
}

\author{
Ahmed Al-Jabir ${ }^{1}$, Abdullatif Aydın', Kamran Ahmed ${ }^{1,2}$, John E. McCabe ${ }^{3}$, M. Shamim Khan ${ }^{1,4}$, \\ Prokar Dasgupta ${ }^{1,4}$, Arun Sahai ${ }^{1,4}$ \\ ${ }^{1}$ MRC Centre for Transplantation, Guy's Hospital, King's College London, London, UK; ${ }^{2}$ Department of Urology, King's College Hospital NHS \\ Foundation Trust, London, UK; ${ }^{3}$ Department of Urology, St Helens and Knowsley Teaching Hospitals NHS Trust, Merseyside, UK; ${ }^{4}$ Department \\ of Urology, Guy's and St. Thomas' NHS Foundation Trust, King's Health Partners, London, UK \\ Contributions: (I) Conception and design: A Aydin, K Ahmed, S Khan, P Dasgupta; (II) Administrative support: JE McCabe, A Al-Jabir; (III) Provision \\ of study materials or patients: JE McCabe, A Sahai; (IV) Collection and assembly of data: A Aydin, K Ahmed, A Al-Jabir; (V) Data analysis and \\ interpretation: A Al-Jabir, A Aydin; (VI) Manuscript writing: All authors; (VII) Final approval of manuscript: All authors. \\ Correspondence to: Dr. Abdullatif Aydın, BSc (Hons), MBBS. MRC Centre for Transplantation, Guy's Hospital, King's College London, London SE1 \\ 9RT, UK. Email: abdullatif.aydin@kcl.ac.uk.
}

Background: The aim of this study is to assess validity of the ETXY Multifunctional trainer (ProDelphus, Olinda, Pernambuco, Brazil), a bench-top dry-lab model for simulation of cystoscopy and intravesical injections of Botulinum Toxin A (BTX-A) injections, in terms of educational value, feasibility and acceptability as well as evaluating the use of fresh frozen cadavers for intravesical BTX-A injections.

Methods: Prospective study with novice trainees and urologists $(n=58)$ trained by experts $(n=14)$ in a 30min hands-on training session in intravesical administration of BTX-A over 6 training sessions throughout one year. Outcome measures were demonstrated through distribution and analysis of evaluation surveys on a 5-point Likert scale.

Results: There were 56 participants (96.6\%) believed that the model has a role in training for the procedure. Participants also reported the training being an important confidence-booster for performing BTX-A injections (mean: 4.05/5) and useful for teaching procedural steps (mean: 3.89). Experts highly rated the realism of the simulator especially in simulation of needle penetration (mean: 3.98 ) and delivery (mean 4.03). Fresh frozen cadavers had a mean realism rating of 4.54 and participants affirmed that they should be routinely used for training and assessment (mean: 3.92).

Conclusions: This study demonstrated face and content validity in addition to establishing the feasibility and acceptability of the ETXY Multifunctional model in the training of intravesical BTX-A administration. Additionally, the simulator demonstrated educational value and fresh frozen cadavers were shown to be the preferred simulation modality for this procedure. Further evaluation in randomised controlled studies is needed to demonstrate higher evidence quality.

Keywords: Urology; simulation; procedural training; Botulinum toxin; BOTOX

Submitted May 23, 2019. Accepted for publication Nov 05, 2019.

doi: $10.21037 /$ tau.2019.11.11

View this article at: http://dx.doi.org/10.21037/tau.2019.11.11

\section{Introduction}

In recent years, advances in technology have revolutionised surgical training methods. The utilisation of virtual reality simulators, bench-top and animal models has started to play a greater role in surgical training $(1,2)$ with increasing numbers of studies supporting and evaluating their use in urology (3). However, further evidence should be gathered for procedure-specific use in a greater number of procedures. This importance is emphasized by the unavailability of cadaveric models, or wet lab based training, 


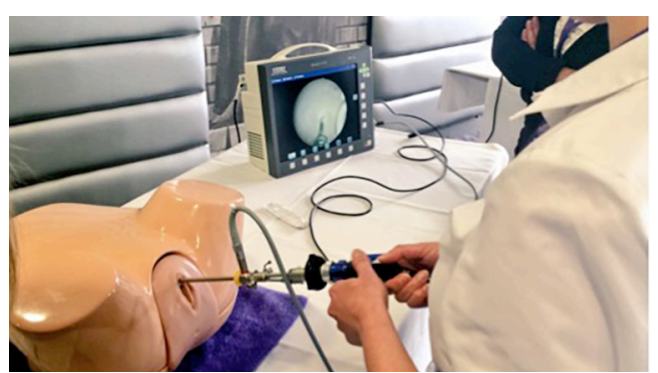

Figure 1 The ETXY Multifunctional Trainer (ProDelphus, Olinda, Pernambuco, Brazil).

and the cost efficiency of implementing alternative to reduce the demand of cadavers (4).

Intravesical injections of Botulinum toxin A (BTX-A) for the treatment of neurogenic detrusor overactivity as well as idiopathic overactive bladder and resulting urge incontinence represents a significant development in management $(5,6)$. However, there is a lack of validated simulation models for clinicians to train in this commonly performed procedure.

The aim of this study is to evaluate the validity of a novel dry-lab model for cystoscopy and BTX-A injections training. It also aims to evaluate the educational value of this training tool and the feasibility and acceptability of incorporating it within training curricula for endourology. Finally, it aims to evaluate the role of cadaveric training for this procedure.

\section{Methods}

\section{Training models utilised}

The ETXY Multifunctional Trainer (ProDelphus, Olinda, Pernambuco, Brazil) is a high-fidelity bench model which includes a changeable male and female urethra and a simulated bladder (Figure 1). This model was utilised in training sessions with a female urethra attached, as is common in real scenarios. A select group of participants also had the opportunity to perform the procedures on fresh frozen cadavers, as part of a full cadaveric training programme (7).

\section{Study process}

Fifty-eight trainees and specialists participated in this prospective study. Participants were all given a theory of the procedure followed by a 30 -min hands-on training session. Each participant performed rigid and/or flexible urethrocystoscopy followed by intravesical BTX-A injections during this time-frame. Sessions were supervised by experts $(n=14)$, who had each performed more than fifty BTX-A procedures. Six training sessions were conducted, three of which also included the use of fresh frozen cadavers. All participants and faculty members were invited to complete an anonymous evaluation survey. Ethics approval was not required for this study.

\section{Outcome measures}

The outcome measures were content and face validity [as defined by McDougal et al. (8)], feasibility, acceptability, perceived educational value and preference of simulation modality. These were measured through the distribution and analysis of evaluation surveys, in line with the current literature base.

\section{Results}

\section{Demographics}

For the purposes of this study 72 subjects were recruited, comprising of 58 trainee-level surgeons and/or BTX-A novices, and 14 experts. Of the 58 novices, 18 were core surgical trainees, 20 urology trainees, 11 gynaecology trainees and 6 nurses (Figure 2). Three participants failed to report their level of proficiency. Only $35.7 \%$ of the novices reported that they had previously received some formal intravesical BTX-A training.

\section{Content and face validity}

Participants completed a number of questions to determine the adequacy of the dry-lab model for training purposes. On a 5-point Likert scale (Figure 3), 56 of the participants believed that the dry lab model has a role in training for the procedure (mean: 4.00/5), that it should be integrated into assessment (mean: 3.89 ) and that it should be incorporated into urological training (mean: 4.22).

Participants were also requested to evaluate how representative the dry-lab model was at training for the procedure (Figure 3). Participants reported the session to be useful for learning procedural steps (mean: 3.89), for familiarisation with relevant anatomy (mean: 3.76) and an important confidence-booster for performing BTX-A injections (mean: 4.05). Fifty-two participants also agreed 


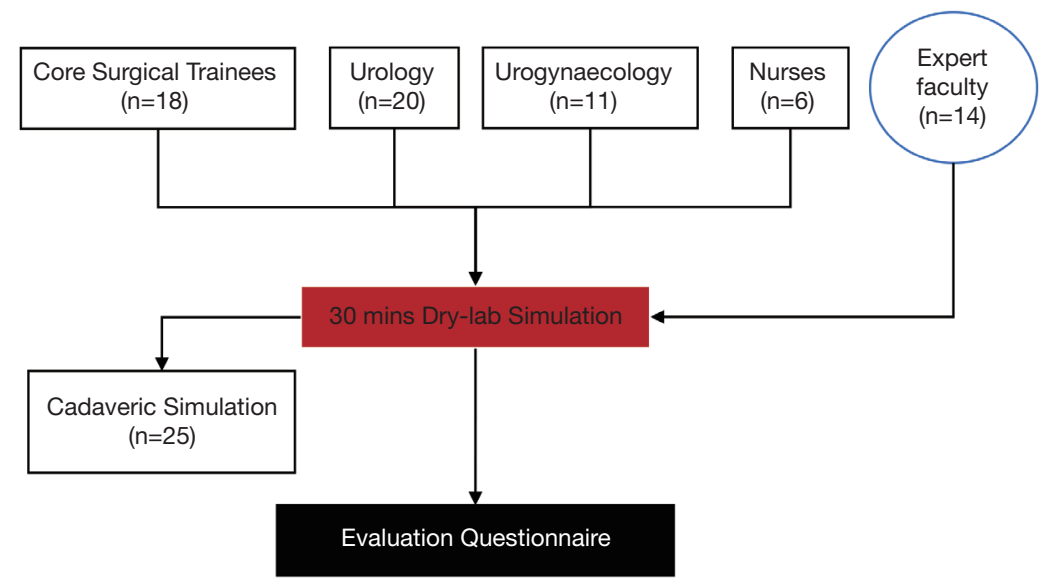

Figure 2 The study process.
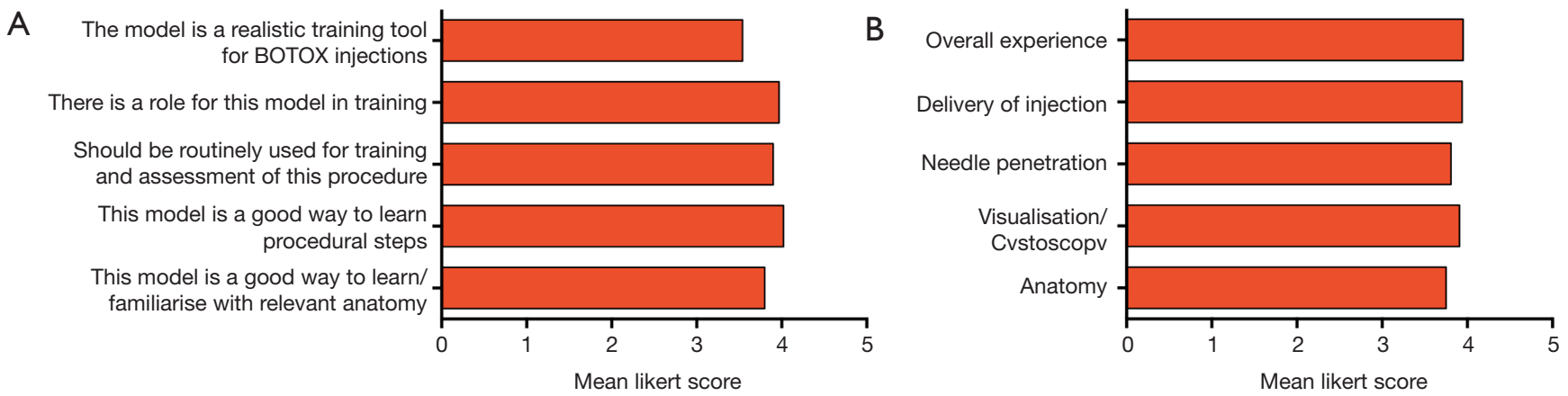

Figure 3 Mean Likert scores of questions relating to (A) content validity and (B) face validity.

that simulation based training helps to improve patient safety (mean: 4.1).

Experts responded to questions regarding similarity real scenarios (mean: 3.64) and whether the model was realistic in terms of anatomy (mean: 3.62), cystoscopy (mean: 3.62), needle penetration (mean: 3.31 ) and delivery of injections (mean: 3.69) on a Likert scale. The overall responses suggested similarity between simulation and reality (mean: 3.95 ) in terms of anatomy (mean: 3.80), cystoscopy (mean: 4.00), needle penetration (mean: 3.98 ) and delivery (mean 4.03).

\section{Feasibility and acceptability}

Participants answered questions regarding the acceptability and feasibility of incorporating simulationbased training within training programs. Simulation-based training and assessment was perceived to be essential for patient safety (mean: 4.15/5) and it was agreed that that there is a role for a validated simulation programme in urology (mean: 4.29). Feasibility results suggested that it is feasible to incorporate this method of training into training programmes (mean: 4.27). However, only $53 \%$ of respondents believed that use of simulation should be part of accreditation/recertification.

\section{Educational value}

Participants believed that the session significantly improved their skills (mean: 4.02/5) and that they gained transferrable skills (mean: 3.95). A significant proportion recommended the training session to others (mean: 4.14). When asked, what the best method for training for BTX-A injections, $87 \%$ responded with supervised simulation followed by operating room training. 


\section{Fresh frozen cadavers}

Experts and 25 participants were asked for the role of wetlab cadaveric training. Fresh frozen cadavers were rated to be realistic for training (mean: 4.54/5) and affirmed that they should be routinely used for training and assessment of these procedures (mean: 3.92). It was also agreed that there is a role for wet-lab training for this procedure (mean: 4.32) and when asked to rate in order of preference, all of the respondents preferred this method of training to be superior than dry-lab simulation.

\section{Discussion}

With the current proliferation of simulation models being developed in the field of urosimulation, there are simulators available for ureteroscopy (9), laparoscopy (10), nephrectomy (11) and robotic surgery (12) but none available for intravesical injection of BTX-A. The current study employed a short simulation session giving teaching with expert supervision on this common procedure and showed performance and high ratings by all disciplines of health professional involved in using BTX-A in clinical practice showing versatility.

Following the established definitions in the literature described by McDougall (8), a survey was used to explore opinions of the realism for the simulation of the intended procedure (face validation). This shows that the ETXY Multifunctional Trainer was rated highly (mean: 3.95/5) and thus meets this domain of validation. Content validation concerns the usefulness of the simulator whether it is appropriate or not. About $80 \%$ believed that this simulator is appropriate for the intended procedure and should be used in the context of simulating intravesical BTX-A administration. Additionally, participants believed that this model should form part of assessment (mean: 3.89) as well as of broader urological training (mean: 4.22). When asked regarding the need for simulation, participants stated there is a clear need for it for the purposes of patient safety (mean: 4.15 ) and that it is feasible to introduce such an element into training programmes (mean: 4.27). Furthermore, participants enjoyed the course and $82.8 \%$ of participants would recommend it to a colleague. Although BTX-A injections are a straight forward simple procedure, there are some tips and tricks to ensure effective delivery of injections such as fullness of the bladder during injections, burying the needle to the hilt before injecting and briefly waiting after the delivery before removing needle. The sessions also gave trainees an opportunity to familiarise with the instrument and consumable kit, essential for cognitive preparation.

The second element of this study focused on the use of fresh frozen cadavers for intravesical BTX-A administration simulation. It was shown to be $90.8 \%$ realistic with a mean Likert rating of $3.92 / 5$ for participants views on routine use in training programme. Significant here is the opinions of the experts and novice participants alike that FFC is the preferred method of simulation, above the ETXY and other dry-lab simulators. This is in line with previous findings in the literature that cadaveric simulation was more highly rated than dry-lab and other wet-lab forms of simulationbased training such as live animals (7).

This study also had some limitations with a heterogenous novice arm comprising of small numbers from each speciality making sub-group analyses statistically unfeasible. Additionally, only a short period of hands-on time $(30 \mathrm{~min})$ was given for each participant to learn the procedure using the ETXY Multifunctional Trainer. Furthermore, due to a limited supply of cadavers, it was not possible to utilise in them in all sessions. Higher-quality randomised controlled trials are needed to provide the best possible evidence for the use of the ETXY Multifunctional trainer.

\section{Conclusions}

This study demonstrated face and content validity in addition to establishing the feasibility and acceptability of the ETXY Multifunctional model in the training of intravesical BTX-A administration. Additionally, the simulator was assessed for its perceived educational value, which was highly rated, and fresh frozen cadavers were shown to be the preferred simulation modality for this procedure. Further research is needed to produce studies of a high level of evidence for the use of the ETXY to train for this procedure as well as consideration of implementation of simulation of intravesical BTX-A as part of a wider simulation based urological curriculum.

\section{Acknowledgments}

The authors acknowledge the support of Allergan in facilitating the running of training sessions and for providing consumables and training models. We thank Dr. Oliver Brunckhorst and Dr. Alexander Khakwani for helping with data collection. A Aydın and P Dasgupta acknowledge support from The Urology Foundation. K Ahmed acknowledges support from The Pelican Foundation. 
P Dasgupta and K Ahmed acknowledge support from the NIHR Biomedical Research Centre, MRC Centre for Transplantation, King's Health Partners, Guy's and St. Thomas' Charity, School of Surgery, London Deanery, Royal College of Surgeons of England, Intuitive Surgical, EU-FP7, Technology Strategy Board and The Vattikuti Foundation.

\section{Footnote}

Conflicts of Interest: Arun Sahai has received an unrestricted educational grant, speaker fees, and is an advisor to Allergan, Ltd. The other authors have no conflicts of interest to declare.

Ethical Statement: The authors are accountable for all aspects of the work in ensuring that questions related to the accuracy or integrity of any part of the work are appropriately investigated and resolved. Ethics approval was not required for this study.

\section{References}

1. Ahmed K, Keeling AN, Fakhry M, et al. Role of virtual reality simulation in teaching and assessing technical skills in endovascular intervention. J Vasc Interv Radiol 2010;21:55-66.

2. Aydin A, Raison N, Khan MS, et al. Simulation-based training and assessment in urological surgery. Nat Rev Urol 2016;13:503-19.

Cite this article as: Al-Jabir A, Aydın A, Ahmed K, McCabe JE, Khan MS, Dasgupta P, Sahai A. The role of dry-lab and cadaveric simulation for cystoscopy and intravesical Botulinum toxin injections. Transl Androl Urol 2019;8(6):673-677. doi: 10.21037/tau.2019.11.11
3. Ahmed K, Jawad M, Abboudi M, et al. Effectiveness of procedural simulation in urology: a systematic review. J Urol 2011;186:26-34.

4. Cornwall J, Stringer MD. The wider importance of cadavers: educational and research diversity from a body bequest program. Anat Sci Educ 2009;2:234-7.

5. Leippold T, Reitz A, Schurch B. Botulinum toxin as a new therapy option for voiding disorders: current state of the art. Eur Urol 2003;44:165-74.

6. Schurch B, Stöhrer M, Kramer G, et al. Botulinum-A toxin for treating detrusor hyperreflexia in spinal cord injured patients: a new alternative to anticholinergic drugs? Preliminary results. J Urol 2000;164:692-7.

7. Ahmed K, Aydin A, Dasgupta P, et al. A novel cadaveric simulation program in urology. J Surg Educ 2015;72:556-65.

8. McDougall EM. Validation of surgical simulators. J Endourol 2007;21:244-7.

9. Al-Jabir A, Aydin A, Abe T, et al. Validation of the Advanced Scope Trainer for Flexible Ureterorenoscopy Training. Urology 2017;110:45-50.

10. Aydin A, Shafi AM, Shamim Khan M, et al. Current Status of Simulation and Training Models in Urological Surgery: A Systematic Review. J Urol 2016;196:312-20.

11. Brewin J, Nedas T, Challacombe B, et al. Face, content and construct validation of the first virtual reality laparoscopic nephrectomy simulator. BJU Int 2010;106:850-4.

12. Whittaker G, Aydin A, Raison N, et al. Validation of the RobotiX Mentor Robotic Surgery Simulator. J Endourol 2016;30:338-46. 